\title{
Reconstruction with a pectoralis major myocutaneous flap after left first rib and clavicular chest wall resection for a metastasis from laryngeal cancer
}

\author{
Francesco Paolo Caronia • Alfonso Fiorelli • \\ Fabio Zanchini - Mario Santini - Attilio Ignazio Lo \\ Monte $\cdot$ Sergio Castorina
}

Received: 12 August 2014/ Accepted: 3 October 2014/Published online: 16 October 2014

(C) The Japanese Association for Thoracic Surgery 2014

\begin{abstract}
We presented a case of recurrent metastasis from epidermoid cancer that occurred in the left clavicle of a patient with a history of laryngeal cancer treated on April 2005 with extended hemilaryngectomy, neck dissection and chemoradiation therapy. On September 2008, he developed a left clavicular metastasis. The disease was initially well controlled by chemoradiotherapy but it recurred 17 months later. The optimal treatment plan was established by several multidisciplinary meetings and the patient subsequently underwent an en bloc resection of the left clavicle, first rib and all the other involved structures. Coverage of the thoracic defect was achieved using pectoralis major myocutaneous flap. The patient had a successful surgical outcome. At 1-year follow-up, he had no evidence of disease, a good cosmetic result and returned to
\end{abstract}

F. P. Caronia and A. Fiorelli contributed equally to this work.

\section{F. P. Caronia}

Istituto Oncologico del Mediterraneo, 7 Via Penninazzo, 95029 Viagrande, Catania, Italy

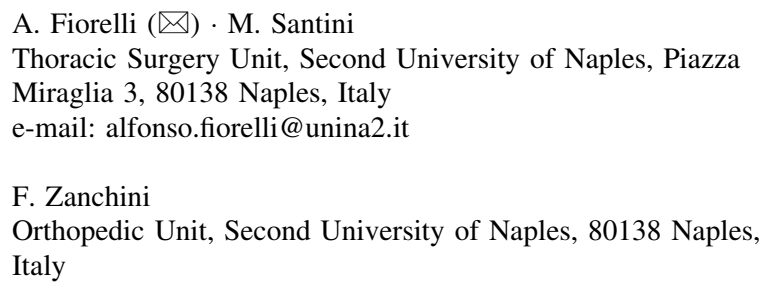

\section{A. I. Lo Monte}

Department of General Surgery, University of Palermo, 129 Via del Vespro, 90127 Palermo, Italy

S. Castorina

G.B. Morgagni Foundation, Department of Bio-Medical

Sciences, University of Catania, Catania, Italy normal daily activity. He died for bone metastasis with an overall 21 months post-surgical survival.

Keywords Chest wall involvement - Chest wall reconstruction · Laryngeal cancer · Muscle flap · Clavicular resection

\section{Introduction}

Surgery of either primary or metastatic chest wall neoplasms is a challenging problem with variable procedure and reconstruction requirements. Herein, we reported a recurrent metastasis of the clavicle secondary to laryngeal cancer successfully resected by multidisciplinary surgical team.

\section{Case}

On April 2005, a 64-year-old male underwent laryngectomy for a squamous cell carcinoma (pathological stage: T2N1M0). He was disease-free until September 2008, when developed metastatic lesion of the left clavicle and first rib treated with chemoradiotherapy. The disease remained well controlled until January 2010 when it progressed. A radiotherapy was started, but lesion progressed with dramatic disability of the left shoulder and limitation of daily living. Computed tomography (CT) scan and 3D reconstruction (Fig. 1) showed that tumor involved the left clavicle, the anterolateral part of the first rib, the sternocostal joint, the left subclavian vein and the overlying skin, while subclavian artery and brachial plexus were freedom from tumor. No other metastasis was found on Position Emission tomography (PET) and bone scintigraphy. For 

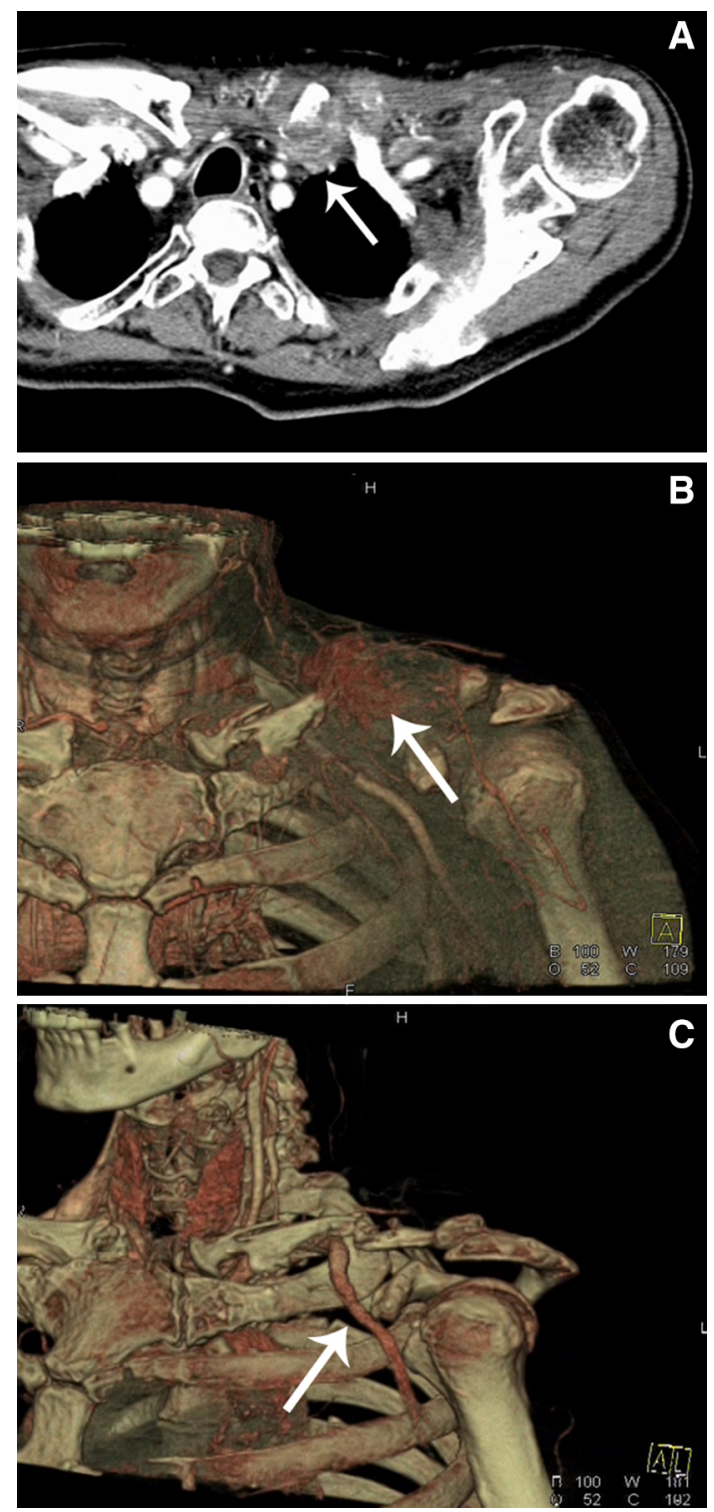

Fig. 1 Computed tomography scan (a) and 3D reconstruction (b) showed a mass that involved the left clavicle and the anterolateral part of the first rib with its sternocostal joint. The subclavian artery and brachial plexus were free from tumor (c)

the extent of his previous radiotherapy doses, the patient developed radiation-induced dermatitis of the left-sided cervical and supraclavicular skin. After multidisciplinary meeting, surgery was proposed as the best treatment obtaining a informed signed consent by patient. He was placed in lateral decubitus with the arm prepped and protected by drapes so that it could be moved within the operative field, as previously described [1]. It allowed a transmanubrial approach and to perform the transposition of any muscle flaps without the need of changing the position of the patient. The procedure, summarized in Fig. 2, began with video-thoracoscopic exploration of the pleural cavity that excluded pleural involvement. Then, a transmanubrial L-shaped incision was performed which allowed retraction of an osteomuscular flap including the left upper portion of the sternal manubrium, the clavicle and all of its muscular insertions. A rib spreader was used to elevate this osteomuscular flap, which allowed access to the neurovascular structures of the thoracic inlet. The incision line was continued laterally toward the deltoid region and posteriorly along the upper border of trapezius muscle to join anteriorly at the upper edge of the L-shaped incision. The dissection continued toward the subclavian vessels and the brachial plexus, which were dissected free from their attachments. The left internal thoracic vessels and subclavian vein were resected, after proximal and distal vessel control, without reconstruction. Anteriorly, the clavicle was removed, including the left upper portion of the manubrium. Posteriorly, it was disarticulated by sectioning the conoid, trapezoid and acromioclavicular ligaments. The clavicle, the entire first rib and all involved structures were removed en bloc. A left pectoralis major myocutaneous flap based medially was transposed upwards and sutured in place to restore the defect, measured $22 \times 18 \mathrm{~cm}$. Chest drains were placed through the thoracoscopic port. Pathological results showed tumor-free resection margins of at least $2 \mathrm{~cm}$. The postoperative course was unremarkable and the patient was discharged on postoperative day 15 . His shoulder was immobilized for 2 months using a shoulder-arm immobilizer; then, he underwent rehabilitation with improvement of shoulder function (Table 1). At the 1-year follow-up, he was well, without tumor recurrence or atrophy of myocutaneous flap. Seventeen months after operation he had a vertebral metastasis and died 4 months later, with an overall 21 months post-surgical survival.

\section{Discussion}

The operative indication for this case may be controversial and contentious. However, it resulted by a multidisciplinary meeting including oncologist, radiologist, radiotherapist, anesthesiologist, thoracic, orthopedic, vascular, and plastic surgeons and physiotherapist. Because (1) the patient have yet received high dose of radiotherapy that contra-indicated further treatments, (2) the bone lesion was the only identifiable site of disease on CT and PET scan; (3) he was fit for surgery, and (4) there was the possibility of improving shoulder function with complete resection of tumor, oncologist and radiotherapist stated surgery as the best treatment for patient. A thoracoscopic exploration of pleural cavity was planned with the aim of performing tumor resection if no pleural involvement was found. Considering the complexity of operation, it was carried out by a multidisciplinary surgical team. Orthopedic surgeon 
Fig. 2 After thoracoscopic exploration of the pleural cavity to exclude pleural involvement, a transmanubrial L-shaped incision was performed (a). The clavicle was disarticulated by sectioning the conoid and trapezoid ligaments (b). The clavicle, the entire first rib and all involved structures were resected en bloc $(\mathbf{c})$. The subclavian vessels and the brachial plexus were free from tumor $(\mathbf{d})$. The defect measured $22 \times 18 \mathrm{~cm}(\mathbf{e})$. A left pectoralis major myocutaneous flap based medially was transposed upwards (f) and sutured in place to restore the defect (g). At the 1-year followup visit, no recurrence or atrophy of the transposed pectoralis major muscle was found (h)
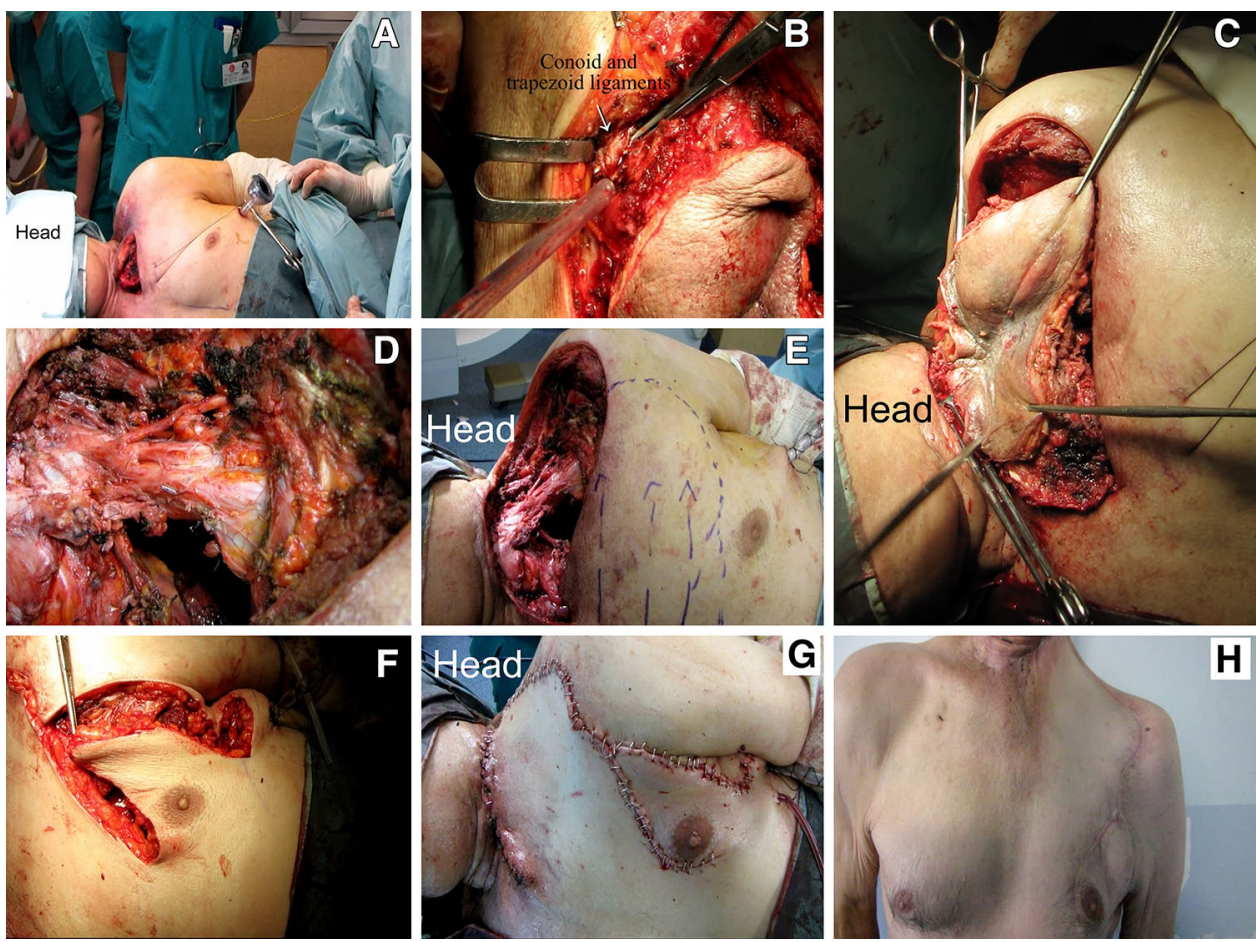

Table 1 Results of the cuff tear function and range of motion tests before and after surgery

\begin{tabular}{lll}
\hline & Preoperative & $\begin{array}{l}\text { One year } \\
\text { postoperatively }\end{array}$ \\
\hline Test di Neer & ++- & ++- \\
Jobe test & +-- & +-- \\
Lag test & --- & --- \\
Bely test & --- & --- \\
VAS & 10 & 2 \\
Forward flexion & $60^{\circ}$ & $90^{\circ}$ \\
Shoulder & $40^{\circ}$ & $70^{\circ}$ \\
abduction & & \\
Internal rotation & $\mathrm{S} 1$ & Iliac crest \\
External rotation & $20^{\circ}$ & $35^{\circ}$ \\
Cross-arm & Impossible due to skin and & Possible \\
$\quad$ horizontal & sternocleidomastoid muscle & \\
adduction test & fibrosis & \\
\hline
\end{tabular}

${ }^{a}$ VAS Visual Analog Scale pain score ranged from 0 (no pain) to 10 (worst imaginable pain) scores

helped to resect clavicular and first rib, vascular surgeon facilitated the dissection of tumor from the neurovascular structures of thoracic inlet, and plastic surgeon restored the defect with myocutaneous flap. Finally, physiotherapist was responsible of postoperative rehabilitation after removing shoulder-arm immobilizer. The "pro et contra" of the different procedures reported in literature for management of similar cases were reviewed by multidisciplinary surgical team to offer patient the best procedure.
An anatomical reconstruction of the clavicle using vascularized osteotomized fibula technique as reported by Kalbermatten et al. [2] was excluded for the high risk of failure due to the previously irradiated region. It is, in fact, common to see bony non-union because of necrotic fibrosis due to radiation for cancer.

Clavicular reconstruction with a compound rib latissimus dorsi [3, 4] or a rib serratus anterior [5] osteomusculocutaneous pedicled flap, using a 4-6 cm long segment of the sixth rib, was also rejected. It would have reduced the posterior stability of the shoulder, and furthermore, it would have necessitated opening the left chest cavity elsewhere.

The use of a myocutaneous flap of pectoralis major to cover chest wall defects is widely reported in the literature with high success rates [6-10]. A pectoralis major myocutaneous flap can be based either on its primary pedicle, the thoraco-acromial artery, or medially, on a vascular supply that originates from perforating branches of the internal thoracic artery, that derive from the fifth to seventh intercostals arteries. Following extensive chest wall resection, the reconstruction technique should fulfill two functional requirements: adequate rigidity and flexibility of the new chest wall during the breathing phases. The myocutaneous pectoralis major flap enables a balance between these two parameters thus favoring the patient's respiratory dynamics and producing low morbidity and a good functional result.

In this case, the choice of such flap was based on three main factors: (1) the patient had significant functional 
disability of the left shoulder due to the combination of tumor involvement in the clavicle and radiotherapyinduced neck fibrosis; (2) the presence of inflexible tissues in the supraclavicular region would have required a wellvascularized, tension-free flap; and (3) the ease with which this kind of flap could be transposed into the clavicular region to minimize morbidity.

The myocutaneous pectoralis major flap survived without any complications; the patient remained alive with a good cosmetic outcome and good performance status 1 year after the repair. The patient was pain-free, without paradoxical chest movement and returned to normal daily activity. Furthermore, Li et al. [11] confirmed that there is no advantage of allograft reconstruction over no reconstruction in terms of the functional outcomes as well as the complication rate. Therefore, our belief that clavicular reconstruction was not justified in this case appears to be supported.

\section{Conclusions}

In challenging cases as the present, where a salvage operation is performed, teamwork is vital. The possible surgical indications may be broadened by capitalizing on the combined specialist knowledge and skills of a multidisciplinary team with the aim of giving the patient the best therapy to prolong life and improve prognosis and quality of life.

Conflict of interest The authors disclose any conflict of interest and no funding for the present paper.

\section{References}

1. Caronia FP, Fiorelli A, Ruffini E, Nicolosi M, Santini M, Lo Monte AI. A comparative analysis of Pancoast tumour resection performed via video-assisted thoracic surgery versus standard open approaches. Interact CardioVasc Thorac Surg. 2014;19: 426-35.

2. Kalbermatten DF, Haug M, Schaefer DJ, Wolfinger E, Schumacher R, Messmer P, Pierer G. Computer aided designed neoclavicle out of osteotomized free fibula: case report. Br J Plast Surg. 2004;57(7):668-72.

3. Yel M, Karalezli MN, Tosun Z, Sezgin S, Savaci N. Osteomuscular flap for clavicular reconstruction: case report. Acta Orthop Traumatol Turc. 2007;41(2):152-4.

4. Devaraj VS, Kay SP, Batchelor AG. Vascularised reconstruction of the clavicle. Br J Plast Surg. 1990;43(5):625-7.

5. Werner CM, Favre P, Van Lenthe HG, Dumont CE. Pedicled vascularized rib transfer for reconstruction of clavicle nonunions with bony defects: anatomical and biomechanical consideration. Plast Reconstr Surg. 2007;120(1):173-80.

6. Arnold PG, Pairolero PC. Chest-wall reconstruction: an account of 500 consecutive patients. Plast Reconstr Surg. 1996;98: 804-10.

7. Cohen M, Ramasastry SS. Reconstruction of complex chest wall defects. Am J Surg. 1996;172:35-40.

8. Mansour KA, Thourani VH, Losken A, et al. Chest wall resections and reconstruction: a 25 year experience. Ann Thorac Surg. 2002;73:1720-6.

9. Chang RR, Mehrara BJ, Hu QY, Disa JJ, Cordeiro PG. Reconstruction of complex oncologic chest wall defects: a 10-year experience. Ann Plast Surg. 2004;52:471-9.

10. Arnold PG, Pairolero PC. Use of pectoralis major muscle flaps to repair defects of anterior chest wall. Plast Reconstr Surg. 1979;63:205-13.

11. Li J, Wang Z, Fu J, Shi L, Pei G, Guo Z. Surgical treatment of clavicular malignancies. J Shoulder Elbow Surg. 2011;20: 295-300. 